Reprod. Nutr. Dévelop., 1984, 24 (3), 239-250.

\title{
Comportement alimentaire, rumination, fermentations réticulo-ruminales et acides gras volatils plasmatiques, comparés chez la chèvre et le mouton ; influence du régime
}

\author{
M. FOCANT
}

Université Catholique de Louvain, Laboratoire de Biochimie de la Nutrition, 3. Place Croix-du-Sud, B-1348 Louvain-la-Neuve, Belgique.

\section{Summary. Effect of diet on eating behaviour and rumination and on rumen and blood VFA} concentrations in sheep and goats : a comparative study.

Two-year old Alpine she-goats $(n=3$ ) and Texel ewes ( $n=3$ ) were compared as to eating behaviour, rumination and volatile fatty acids (VFA) in rumen and blood. The animals were fed once daily with two different proportions $(20$ and $80 \%)$ of barley and hay. Dry matter intake was fixed at $48 \mathrm{~g} \mathrm{D.M} / \mathrm{kg} \mathrm{P}^{0.75}$ per day.

In similar feeding and environmental conditions, eating behaviour and rumination of goats and sheep did not differ much : the goats tended to eat faster and there were more rumination periods in the sheep. Latency time, mean duration of rumination periods, daily rumination time and circadian pattern of rumination did not differ significantly between the two species (fig. 1).

With both diets we observed a higher VFA concentration and a lower acetate/propionate ratio in the rumen of the ewes; however, rumen $\mathrm{pH}$ was lower only in those eating the $80 \%$ barley ration (fig. 2 ).

Blood VFA in the jugular vein did not differ between sheep and goats (fig. 3). The proportion of cereals and hay in the diets affected rumen fermentation and rumination pattern in both species. With a higher concentrate/roughage ratio, rumen and plasma VFA increased, while the $\mathrm{pH}$ and acetate/propionate ratio in the rumen juice, the number of rumination periods and daily rumination time decreased. When the animals were fed the $80 \%$ barley ration, there was practically no rumination in the first $9 \mathrm{~h}$ after the single meal. During this time, rumen $\mathrm{pH}$ was minimal and VFA levels in the rumen and blood were maximal.

\section{Introduction.}

II y a relativement peu de travaux de physiologie digestive réalisés sur la chèvre. Wainman (1977) écrivait qu'on ne peut pas considérer un taurillon comme un gros mouton ; il en est de même pour la chèvre qui se distingue de la brebis par de nombreux aspects. Pourtant peu de comparaisons ont été menées entre ani- 
maux d'espèces différentes, dans des conditions physiologiques et d'environnement identiques (Dulphy et al., 1980). En particulier, le comportement alimentaire et mérycique des caprins et des ovins a été peu comparé (Ruckebusch, 1963 ; Geoffroy, 1974).

Par contre, I'utilisation digestive comparée des aliments a été plus étudiée. Les caprins consomment davantage que les ovins : leurs besoins en énergie métabolisable pour l'entretien sont plus grands (Mohamed et Owen, 1981). La littérature relate deux types de résultats. Les premiers ont trait à l'utilisation digestive des fourrages communément distribués dans les régions tempérées et montrent une égale capacité de la chèvre et du mouton à digérer la cellulose (Jones et al., 1972 ; El-Hag, 1976 ; Blanchart et al., 1980; Mohamed et Owen, 1981). Les autres concernent des fourrages tropicaux pauvres et mènent à la conclusion que la chèvre digère mieux les fibres que le mouton (El-Hag, 1976; Gihad, 1976 ; Gihad et al., 1980 ; Devendra, 1978, 1981). Toutefois, Van Soest (1982) attribue ces derniers résultats à la nature même des fourrages tropicaux qui sont formés d'herbes ayant des valeurs nutritives fort différentes et qui offrent la possibilité à la chèvre de sélectionner les parties les plus digestes de son aliment, faculté que n'a pas le mouton.

Devendra (1981), de son côté, explique logiquement ses résultats par un temps de rétention $\left(\mathrm{Cr}_{2} \mathrm{O}_{3}\right)$ plus long chez ses chèvres. Nous pensons donc, comme Gihad et al. (1981), que la plus grande efficience digestive des caprins visà-vis des fourrages pauvres est probablement l'apanage de certaines races faibles productrices, vivant dans des environnements difficiles, et n'est certainement pas une caractéristique valable pour toutes les populations caprines!

Ce travail a pour but de comparer le comportement alimentaire, la rumination et les fermentations réticulo-ruminales de la chèvre et de la brebis avec deux rations de niveaux énergétiques très différents, dans des conditions d'alimentation et d'environnement identiques.

\section{Matériel et méthodes.}

Trois chèvres de race Alpine chamoisée pesant entre 45 et $50 \mathrm{~kg}$ et trois brebis de race Texel de 40 à $50 \mathrm{~kg}$ ont été utilisées. Les 6 animaux âgés de 2 ans n'étaient ni en gestation ni en lactation, et ils portaient une canule au niveau du sac dorsal du rumen depuis plus de 6 mois. Pendant tout ce temps, ils ont vécu côte à côte attachés dans des loges individuelles aménagées dans une pièce leur étant réservée, isolée du bruit et de la luminosité extérieure. La température a été maintenue entre 18 et $20^{\circ} \mathrm{C}$, tandis que l'éclairage artificiel a dispensé sa lumière de 7 à $19 \mathrm{~h}$.

Chaque animal a reçu une ration individuelle calculée en fonction de son poids métabolique $\left(\mathrm{kg} \mathrm{P}^{0,75}\right)$ et fixée à $48 \mathrm{~g} \mathrm{MS} / \mathrm{kg} \mathrm{P}^{0,75}$ par jour. Dans une première période expérimentale, la ration était composée de $80 \%$ d'un bon foin de prairie et de $20 \%$ d'orge aplatie, tandis que les pourcentages étaient inversés dans une seconde période. Les aliments furent distribués en un repas unique à $9 \mathrm{~h}$ le matin. L'orge étant toujours consommée en moins de $20 \mathrm{~min}$, le foin était 
donné après. En outre, l'eau et le sel à lécher étaient disponibles à volonté. Chaque période expérimentale était constituée de 4 jours pendant lesquels les mouvements de mastication étaient enregistrés en permanence, selon la méthode de Ruckebusch (1963), et d'un $5^{e}$ jour au cours duquel des échantillons de jus de rumen et de sang ( $\mathrm{v}$. jugulaire) étaient prélevés à $9 \mathrm{~h}$ avant le repas et à intervalles de $3 \mathrm{~h}$ jusqu'à $18 \mathrm{~h}$ (T0, T3, T6, T9). Les animaux ayant été alimentés plusieurs mois avec du foin et de l'orge, 2 semaines d'adaptation furent jugées suffisantes avant la première période expérimentale. Après celle-ci, une semaine de transition progressive permit aux animaux $d$ 'ingérer sans problème une ration renfermant $80 \%$ d'orge et la seconde période de mesure débuta après une nouvelle semaine d'adaptation.

L'enregistrement de la mastication a permis le calcul des paramètres du comportement alimentaire (durées et vitesses d'ingestion) et mérycique (nombre et durée des périodes de rumination, répartition nycthémérale, durée unitaire de rumination). Les acides gras volatils ont été analysés par chromatographie en phase gazeuse après défécation alcoolique pour le sang (Rémésy, 1973) et à l'acide métaphosphorique pour le liquide ruminal.

\section{Résultats et discussion.}

\section{Ingestion.}

Les quantités ingérées étaient fixées à $48 \mathrm{~g} \mathrm{MS} / \mathrm{kg} \mathrm{P}^{0,75}$ par jour et consommées en totalité entre $9 \mathrm{~h}$ et $12 \mathrm{~h}$ le matin. En conséquence, les seuls paramètres de l'ingestion que nous pouvions étudier étaient les durées unitaires d'ingestion ( $\mathrm{min} / \mathrm{g} \mathrm{MS}$ ing. $/ \mathrm{kg} \mathrm{P}^{0,75}$ ) de l'orge et du foin, et la consommation d'eau (voir tableau 1$)$.

TABLEAU 1

Valeurs des paramètres de l'ingestion en fonction du régime et de l'espèce.

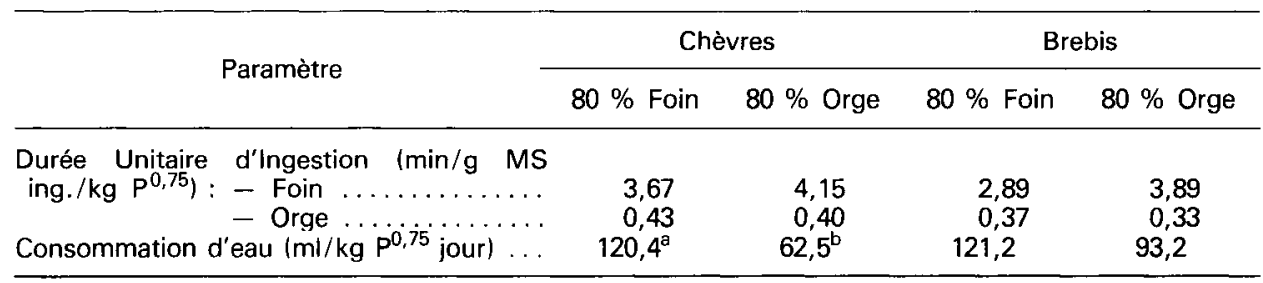

Les moyennes suivies de lettres distinctes diffèrent significativement $(P<0,05)$.

La durée unitaire d'ingestion du foin est près de 10 fois plus élevée que celle de l'orge. II s'agit là d'un phénomène très connu qui ne mérite plus qu'on s'y attarde. Les vitesses d'ingestion ont tendance à être plus lentes chez les chèvres que chez les brebis mais la différence n'est pas statistiquement significative. Ruckebusch (1963) avait déjà remarqué que la brebis consommait son foin de façon 
plus régulière et plus méthodique que la chèvre. Cette constatation fut confirmée par Geoffroy (1974) qui enregistra des DUI (durées unitaires d'ingestion), plus faibles chez le mouton pour l'ingestion de foin et d'ensilages distribués ad libitum. En fait, lorsqu'elle est nourrie à volonté, la chèvre a davantage le loisir de sélectionner son aliment car, friande des feuilles, elle aime faire un tri, ce qui ralentit sa vitesse d'ingestion. La consommation d'eau n'est pas significativement différente $d^{\prime} u n e$ espèce à l'autre pour chacun des régimes. Par contre, l'eau de boisson est absorbée en moins grande quantité avec la ration à $80 \%$ d'orge. Cette différence liée au régime $n$ 'est cependant significative que pour les chèvres $(P<0,05)$. La moindre consommation d'eau avec la ration la plus riche en orge doit sans doute être mise en relation avec la diminution linéaire du " turnover " et du " rumen fluid volume " enregistrée par Huntington et al. (1981), lorsqu'ils augmentaient la proportion de concentrés dans leur régime.

\section{Rumination.}

Tous les paramètres du comportement mérycique sunt présentés dans le tableau 2, tandis que le tableau 3 expose les résultats de l'analyse de la variance relatifs à ceux-ci.

TABLEAU 2

Variations des paramètres de la rumination en fonction du régime alimentaire et de l'espèce.

\begin{tabular}{|c|c|c|c|c|}
\hline \multirow{2}{*}{ Paramètre } & \multicolumn{2}{|c|}{ Chèvres } & \multicolumn{2}{|c|}{ Brebis } \\
\hline & $80 \%$ Foin & $80 \%$ Orge & $80 \%$ Foin & $80 \%$ Orge \\
\hline \multirow{6}{*}{$\begin{array}{l}\text { Temps de latence }(\min ) \ldots \ldots \ldots \ldots \ldots \ldots \\
\text { Nombre de périodes de rumination } \ldots \ldots \ldots \\
\text { Durée moyenne des périodes }(\min ) \ldots \ldots \ldots \\
\text { Durée quotidienne de rumination }(\min ) \ldots \ldots \\
\text { Durée Unitaire de Rumination du foin } \\
\left.\text { (min } / \mathrm{g} \text { ing. foin } / \mathrm{kg} \mathrm{P}^{0,75}\right) \ldots \ldots \ldots \ldots \ldots \ldots\end{array}$} & $199,7^{\mathrm{ab}}$ & $312,3^{\mathrm{ab}}$ & $156,0^{a}$ & $368,0^{b}$ \\
\hline & $10,1^{\mathrm{ab}}$ & $8,0^{\mathrm{a}}$ & $11,8^{b}$ & $10,2^{\mathrm{ab}}$ \\
\hline & 49,9 & 39,6 & 40,3 & 32,6 \\
\hline & $474,0^{\mathrm{a}}$ & $291,7^{\mathrm{b}}$ & $467,7^{\mathrm{a}}$ & $312,0^{b}$ \\
\hline & & & & \\
\hline & $13,1^{\mathrm{a}}$ & $35,4^{\mathrm{b}}$ & $12,2^{\mathrm{a}}$ & $32,6^{b}$ \\
\hline
\end{tabular}

Les moyennes suivies d'une même lettre ne diffèrent pas significativement $(P<0,05)$.

TABLEAU 3

Résultats de l'analyse de la variance des paramètres de la rumination.

\begin{tabular}{|c|c|c|c|}
\hline Paramètre & Effet espèce & Effet régime & Interaction \\
\hline Temps de latence & N.S. & * & N.S. \\
\hline Nombre de périodes de rumination & $*$ & * & N.S. \\
\hline Durée moyenne des périodes & N.S. & N.S. & N.S. \\
\hline Durée quotidienne de rumination . & N.S. & $* * *$ & N.S. \\
\hline Durée Unitaire de rumination & N.S. & *** & N.S. \\
\hline
\end{tabular}

N.S. = non significatif $;{ }^{*}=\mathrm{P}<0,05 ;{ }^{* * *}=\mathrm{P}<0,001$. 
2.1. Temps de latence. - Nous appelons "temps de latence »le temps séparant le début de la première période de rumination de la distribution de l'aliment (" lag time » de Pearce, 1965). II n'est pas significativement différent pour les chèvres et les brevis, mais la ration la plus riche en céréales provoque un accroissement important $(P<0,05)$ du temps de latence. Lorsque les animaux reçoivent le régime à base de foin, ils commencent à ruminer peu de temps après la fin de leur repas. Par contre, quand on remplace le foin par de l'orge, les temps de latence sont compris, en moyenne, entre 5 et $6 \mathrm{~h}$. De telles variations peuvent avoir plusieurs explications. Tout d'abord, le réflexe de rumination est stimulé et dépend de la présence de matériel abrasif dans le réticulo-rumen (Schalk et Amadon, 1928 ; Welch et Smith, 1971). Le régime à base d'orge contenant nettement moins de fibres que celui à base de foin, il serait logique de penser qu'il a un moindre effet stimulant sur la rumination qui s'en trouverait ainsi retardée. Mais même si elle est réduite avec la ration à $80 \%$ d'orge, nous pouvons supposer que la stimulation du comportement mérycique est maximale juste à la fin du repas. Dès lors, l'existence d'un temps de latence fortement allongé serait contraire au concept exclusif de la rumination provoquée par la présence de fibres dans le rumen (Pearce, 1965). II semble donc raisonnable de postuler l'existence de facteurs inhibiteurs de la mastication mérycique, liés à la fermentation réticuloruminale. La rumination pourrait être inhibée par le taux sanguin d'acides gras volatils (Ruckebusch, 1967 ; Focant et al., 1979), par l'acidité du contenu ruminal ou sa pression osmotique élevée (Welch, 1982).

2.2. Nombre et durée moyenne des périodes de rumination. - Nous avons considéré que la période de rumination devait avoir une durée minimale de 5 min et être séparée d'une autre période par au moins $20 \mathrm{~min}$. Cette définition a été établie à la suite d'une étude antérieure (Focant, non publié) au cours de laquelle nous avons observé que la probabilité pour que la rumination reprenne restait élevée dans les $20 \mathrm{~min}$ suivant son arrêt.

Le nombre de périodes de rumination est plus grand pour la brebis que pour la chèvre $(P<0,05)$. II est plus petit après l'ingestion du régime à base d'orge $(P<0,05)$. D'autre part, la durée moyenne de ces périodes est plus courte pour la brebis et pour la ration la plus riche en céréales, mais ces différences ne sont pas statistiquement significatives. Geoffroy (1974) n'avait pas observé de différences entre espèces quant au nombre et à la durée moyenne des périodes de rumination.

2.3. Durée quotidienne de rumination. - Nous n'observons pas de différence entre chèvres et brebis pour la durée quotidienne de rumination.

La durée de rumination par $24 \mathrm{~h}$ est fortement influencée par le régime $(P<0,001)$. De $8 \mathrm{~h}$ avec la ration à $80 \%$ de foin, le temps de rumination est réduit à $5 \mathrm{~h}$ lorsque la proportion de foin ingéré tombe à $20 \%$. Cela n'est assurément pas un résultat inédit. Il est évident, d'une part, qu'un état plus grossier des aliments provoque une augmentation de la fréquence et de la force des contractions du sac rumino-réticulaire (Ruckebusch, 1967) et, d'autre part, que l'ingestion d'une quantité plus grande de foin va de pair avec une durée plus longue de rumination aussi bien chez le mouton que chez la vache (Bae et al., 1979 et 1981). 
2.4. Durée unitaire de rumination. - La durée unitaire de rumination (DUR) exprime la durée de remastication rapportée à la quantité d'aliments ingérée ( $\mathrm{min} / \mathrm{g} \mathrm{MS}$ ing. $/ \mathrm{kg} \mathrm{P}^{0.75}$ ). Etant donné que la prise alimentaire était fixée et égale pour tous les animaux et pour les 2 régimes, la DUR rapportée à la ration totale ne nous apporte aucun enseignement supplémentaire autre que ceux donnés par la durée quotidienne de rumination. Mais le but primordial du comportement mérycique est de réduire les ingesta en particules suffisamment fines pour passer l'orifice réticulo-omasal. II était donc intéressant de rapporter le temps de rumination aux quantités ingérées de foin.

Si la DUR du foin n'est pas différente entre espèces, elle est fortement influencée par le régime $(P<0,001)$. De $33-35 \mathrm{~min} / \mathrm{g}$ foin ing. $/ \mathrm{kg} \mathrm{P}^{0,75}$ avec la ration contenant $20 \%$ de foin, elle passe à $12-13 \mathrm{~min} / \mathrm{g}$ foin ing. $/ \mathrm{kg} \mathrm{P} \mathrm{P}^{0,75}$ quand la proportion de foin dans le régime est de $80 \%$. Cette diminution de la DUR du foin lorsque son niveau d'ingestion augmente est en accord avec les résultats obtenus chez le bélier et chez la vache par Bae et al. $(1979,1981)$.

2.5. Répartition nycthémérale de la rumination. - La répartition nycthémérale de la rumination par tranche de $3 \mathrm{~h}$ (fig. 1) n'est pas significativement différente pour les chèvres et les brebis. Par contre, I'analyse de la variance met en évidence un effet " heure » et un effet " régime » $(P<0,001)$.

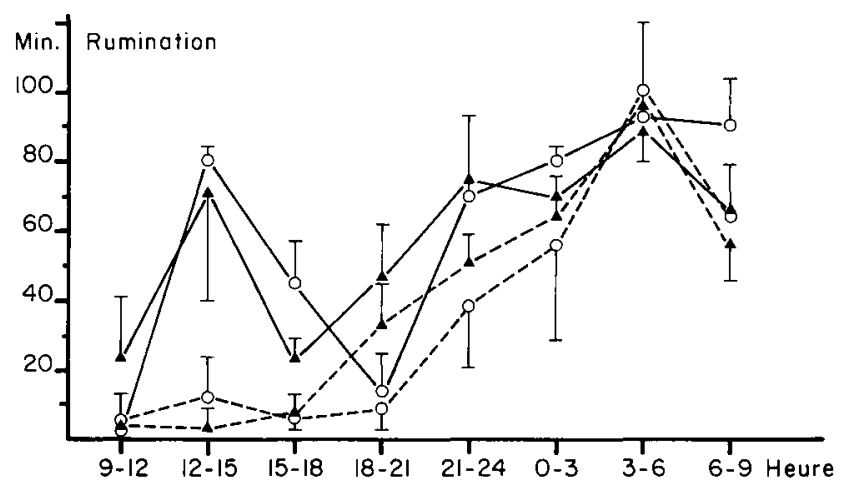

FIG. 1. - Répartition nycthémérale de la rumination en fonction de l'espèce et du régime (moyennes de 3 heures et écart-types). ( $\Lambda$ : brebis ; $:$ : chèvres ; - $80 \%$ foin ; ---- $80 \%$ orge).

Lorsqu'ils reçoivent la ration à $80 \%$ de foin, les animaux ont deux périodes de rumination intenses : de $12 \mathrm{~h}$ à $15 \mathrm{~h}$, et de $21 \mathrm{~h}$ au repas du matin. Le remplacement du foin par l'orge dans le régime modifie le comportement mérycique qui est quasiment absent pendant toute la période diurne suivant le repas. La rumination ne débute qu'à l'extinction des lumières et augmente jusqu'à une durée maximale entre $3 \mathrm{~h}$ et $6 \mathrm{~h}$ le matin. L'influence du régime est manifeste pendant la phase éclairée du nycthémère, mais elle disparaît progressivement après la fermeture de l'éclairage. II n'est pas exclu que l'obscurité soit alors le stimulant principal pour la mastication mérycique (Gordon, 1958b). D'ailleurs, cette rumination intense enregistrée pendant la seconde moitié de la nuit chez nos chèvres et nos 
brebis se retrouve chez tous les ruminants domestiques, quelles que soient leurs conditions d'alimentation (Bell et Lawn, 1957 ; Gordon, 1958a, 1958b ; Pearce, 1965 ; Geoffroy, 1974 ; Metz, 1975 ; Bae et al., 1979).

3. Fermentations dans le rumen (fig. 2).
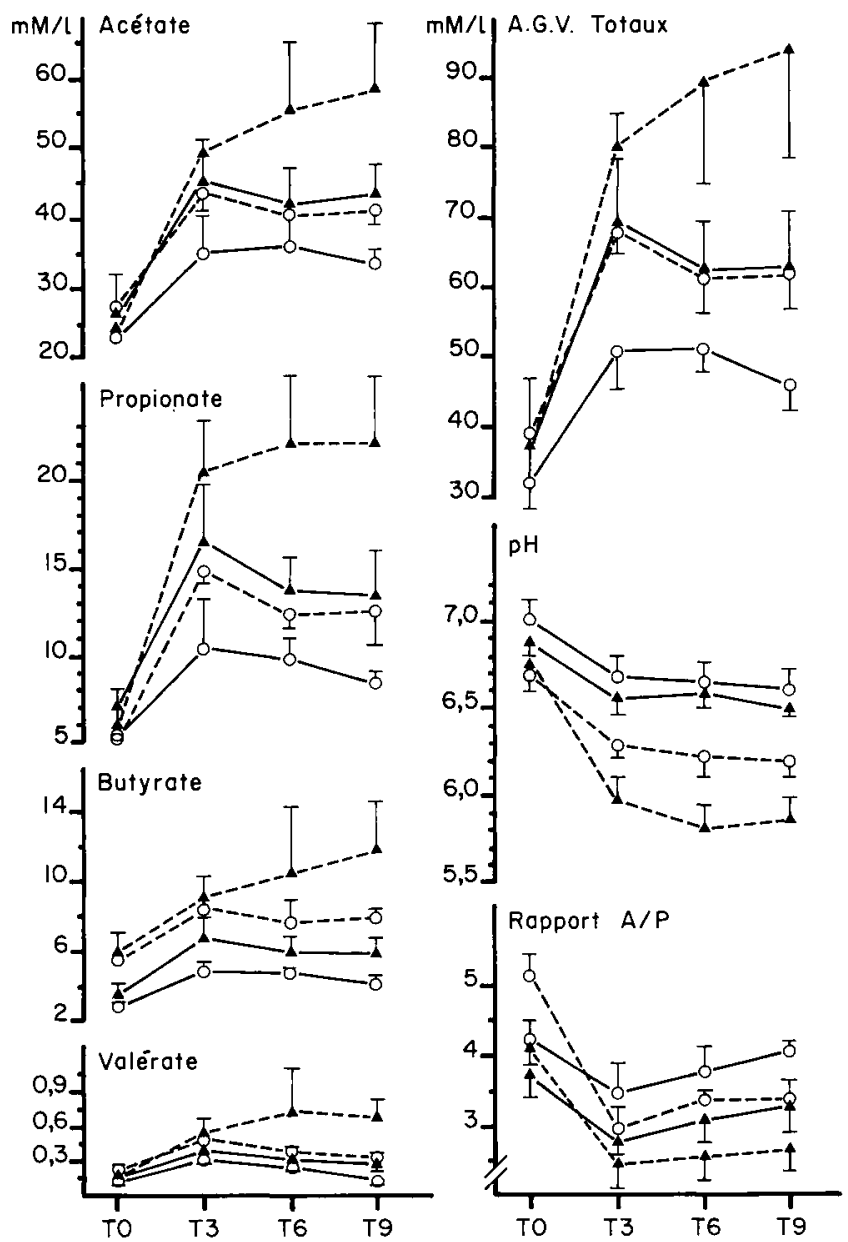

FIG. 2. - Acides gras volatils, $\mathrm{pH}$ et rapport acétate/propionate dans le contenu du rumen en fonction du temps après le repas, du régime et de l'espèce (moyennes et écart-types). (A : brebis ; 0 : chèvres ; -_ $80 \%$ foin ; --- $80 \%$ orge).

3.1. $\mathrm{pH}$ du contenu réticulo-ruminal. - L'analyse statistique des mesures du $\mathrm{pH}$ révèle 3 effets significatifs $(\mathrm{P}<0,001)$ : celui de l'heure du prélèvement, celui du régime et celui de l'espèce. Pour chaque régime et chaque espèce, le $\mathrm{pH}$ enregistré $3 \mathrm{~h}$ après le repas est inférieur à celui mesuré avant l'affouragement, et il continue de décroître jusqu'à T6. Quelle que soit l'heure envisagée, le pH n'est 
pas significativement différent pour les 2 espèces lorsqu'elles sont alimentées avec la ration à $80 \%$ de foin. Au contraire, une alimentation à base de concentrés provoque chez la brebis une acidité plus grande du contenu du rumen que chez la chèvre. Après le repas, le $\mathrm{pH}$ du jus de rumen est toujours plus bas chez les petits ruminants ayant ingéré la ration la plus riche en orge. Par contre, à T0, l'acidité du contenu ruminal n'est modifiée par le régime que chez la chèvre.

3.2. AGV dans le rumen. - La concentration de chaque acide gras volatil pris individuellement est significativement $(P<0,001)$ influencée par l'heure de prélèvement, le régime et l'espèce. Mais comme ils suivent tous la même évolution, l'analyse de la teneur en AGV totaux suffit pour en retirer les enseignements recherchés.

Le repas provoque une élévation importante du taux d'acides gras à courte chaîne qui est d'autant plus grande que le régime est riche en concentrés. Trois heures après la distribution de la ration à $80 \%$ d'orge, on mesure chez la brebis une concentration d'AGV de I'ordre de $80 \mathrm{mM} / \mathrm{I}$ alors qu'au départ, à T0, elle était de $38 \mathrm{mM} / \mathrm{l}$. Avec le régime pauvre en concentrés, la teneur à T3 n'est que de $70 \mathrm{mM} / \mathrm{I}$ avec un même taux initial. Dans la plupart des cas, les concentrations maximales sont enregistrées $3 \mathrm{~h}$ après l'affouragement et elles restent plus ou moins constantes pendant $6 \mathrm{~h}$. Toutefois, chez la brebis alimentée de $80 \%$ d'orge, la teneur en AGV continue d'augmenter jusqu'à T9.

Après un même repas, la concentration réticulo-ruminale d'AGV est plus élevée chez la brebis que chez la chèvre. Neuf heures après le début du repas à $80 \%$ de foin, les teneurs en $A G V$ totaux sont de $46 \pm 4 \mathrm{mM} / \mathrm{l}$ contre $63 \pm 8 \mathrm{mM} / \mathrm{l}$ pour les chèvres et les brebis respectivement. Quand on remplace le foin par l'orge, ces teneurs deviennent $62 \pm 5 \mathrm{mM} / \mathrm{l}$ contre $94 \pm 15 \mathrm{mM} / \mathrm{l}$. L'influence de l'espèce sur les taux d'AGV s'atténue avec le temps puisqu'elle devient nulle à T24 ou T0.

Pour les deux espèces étudiées, le taux d'AGV dans le rumen est toujours plus élevé après l'ingestion de la ration la plus riche en concentrés, c'est-à-dire la plus énergétique. Toutefois, la différence entre régimes décroît au cours du temps puisqu'elle devient nulle après $24 \mathrm{~h}$ (TO). Cela est en accord avec les résultats obtenus par Sudweeks (1977) chez des taurillons de race Jersey et Guernesey.

3.3. Rapport acétate/proprionate. - Nous constatons que pour un même régime, le rapport $A / P$ est toujours plus élevé chez la chèvre que chez la brebis $(P<0,001)$. Ces résultats sont en accord avec ceux obtenus par Fahmy et al. (1977) pour des fourrages de faible qualité. Pour chacune des espèces, il est d'autant plus bas que la proportion d'orge dans la ration est plus grande $(P<0,05)$. Enfin, ce rapport $A / P$ évolue dans le temps en fonction du repas $(\mathrm{P}<0,001)$. Il est minimum $3 \mathrm{~h}$ après l'affouragement et augmente ensuite progressivement jusqu'au repas suivant. Sudweeks (1977) avait obtenu des résultats semblables en augmentant la proportion de concentrés dans la ration de bovins.

Par ailleurs, les différences marquées de $\mathrm{pH}$, de concentration en AGV et du rapport $A / P$ entre ovins et caprins pourraient être liées à des volumes différents des contenus rumino-réticulaires, à leurs populations microbiennes (Gihad et al., 
1980) ou à des temps de rétention plus faibles chez la chèvre (Uden, 1978). L'étude de ces paramètres serait la suite logique de ce travail.

\section{AGV plasmatiques (fig. 3).}

Au niveau de la veine jugulaire, la majeure partie des acides gras volatils est constituée par l'acétate qui varie entre $0,42 \pm 0,03 \mathrm{mM} / \mathrm{l}$ et $1,07 \pm 0,04 \mathrm{mM} / \mathrm{l}$ de plasma. Le taux de propionate est faible et varie de 0,01 à $0,03 \mathrm{mM} / \mathrm{l}$. Le butyrate n'est présent que sous forme de traces qui sont difficilement quantifiables (Simkins et al., 1966).

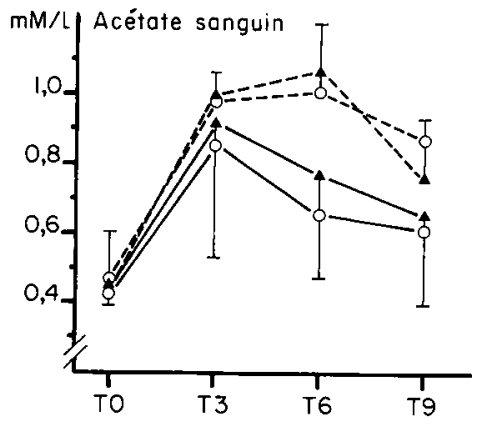

FIG. 3. - Acétate plasmatique en fonction du temps après le repas, du régime et de l'espèce (moyenne et écart-types). (A : brebis ; 0 : chèvres ; - $80 \%$ foin ; ---80\% orge).

La vitesse d'absorption des AGV au niveau de la paroi ruminale étant proportionnelle à leur concentration (Schambye et Phillipson, 1949), nous nous attendions à trouver davantage d'acétate dans le sang des brebis que dans celui des chèvres, proportionnellement aux différences observées dans le rumen. Si tel est le cas lorsque les animaux reçoivent la ration à $80 \%$ de foin (bien que la différence ne soit pas significative, $P>0,05)$, il n'en est rien avec l'alimentation à base d'orge. Les taux d'acétate plasmatique ne sont pas différents pour les deux espèces, alors que la concentration d'acétate dans le rumen des brebis est nettement plus élevée que celle des chèvres. Ce fait pourrait s'expliquer par une vitesse d'absorption des $A G V$ du rumen plus rapide chez la chèvre, ou par une vitesse de métabolisation plus grande de l'acétate sanguin par les tissus de la brebis, à moins que la chute plus brutale du pH ruminal de la brebis, associée à une concentration plus élevée d'acides organiques dans le rumen après l'ingestion du régime riche en concentrés, ne provoque une stase plus importante du réticulorumen, (Ash, 1959). En conséquence, le contenu ruminal serait moins brassé et les AGV moins en contact avec la paroi. La vitesse d'absorption serait aussi ralentie et nous assisterions à une accumulation des AGV dans le rumen (fig. 2). Cette dernière hypothèse aurait donc l'avantage d'expliquer, d'une part, l'évolution différente de la concentration d'AGV dans le rumen chez les deux espèces et, d'autre part, l'absence de relation entre acétate dans le rumen et dans le sang des brebis.

L'influence du repas sur le taux plasmatique d'acétate est manifeste $(P<0,001)$. Quels que soient l'espèce ou le régime envisagé, la concentration mesurée $3 \mathrm{~h}$ après l'affouragement est toujours nettement supérieure au taux ini- 
tial. A T0, la teneur moyenne en acétate est de $0,45 \mathrm{mM} / \mathrm{l}$ tandis qu'à T3, elle est de $0,94 \mathrm{mM} / \mathrm{l}$, soit plus du double.

L'effet régime $(P<0,001)$ est surtout observé $6 \mathrm{~h}$ après le début du repas. Avec la ration à base de foin, la concentration sanguine d'acétate est maximale à T3 et diminue ensuite. Par contre, lorsque le régime à $80 \%$ d'orge est distribué, le taux plasmatique d'acétate augmente jusqu'à T6.

\section{Conclusion.}

Pour des conditions d'alimentation et d'environnement identiques, les comportements alimentaire et mérycique de la chèvre et de la brebis diffèrent peu : la vitesse d'ingestion des caprins semble plus lente et le nombre de périodes de rumination par $24 \mathrm{~h}$ serait plus grand chez les ovins. Par ailleurs, les temps de latence, la durée des périodes, la durée quotidienne, la durée unitaire et la répartition nycthémérale de la rumination ne sont pas significativement différents pour les deux espèces.

Chèvres et brebis se distinguent davantage par leurs fermentations réticuloruminales.

Pour un même régime alimentaire, nous mesurons des concentrations d'AGV plus élevées et un rapport acétate/propionate plus faible dans le rumen des brebis. Mais le $\mathrm{pH}$ du liquide ruminal des ovins $\mathrm{n}^{\prime}$ est plus bas que celui des caprins que lorsque les animaux reçoivent la ration à base de concentrés.

Les teneurs en AGV plasmatiques ne sont pas significativement différentes entre les deux espèces.

Le régime alimentaire influence profondément les fermentations ruminales et le comportement mérycique des petits ruminants. Lorsque la proportion de concentrés augmente dans la ration, les concentrations d'AGV dans le rumen et dans le sang s'élèvent, le $\mathrm{pH}$ et le rapport $\mathrm{A} / \mathrm{P}$ du liquide ruminal, le nombre de périodes et la durée quotidienne de rumination diminuent. Quand les animaux reçoivent le régime à $80 \%$ d'orge, la mastication mérycique est pratiquement absente pendant les $9 \mathrm{~h}$ suivant le repas. $\mathrm{C}^{\prime}$ est alors que le $\mathrm{pH}$ ruminal est minimum et que les concentrations d'AGV dans le rumen et dans le sang sont maximales.

Reçu en juillet 1983.

Accepté en décembre 1983.

Remerciements. - Nous remercions le Dr. P. Wittouck du laboratoire de physiologie des animaux domestiques (UCL) pour son aide logistique efficace.

Ce travail a été réalisé avec les subsides de I'Institut pour I'Encouragement de la Recherche Scientifique dans I'Industrie et I'Agriculture (IRSIA), rue de Crayer, 6 B-1050 Bruxelles. 


\section{Références}

ASH R. W., 1959. Inhibition and excitation of reticulo-rumen contractions following the introduction of acids into the rumen and abomasum. J. Physiol., 147, 58-73.

BAE DANG HO, WELCH J. G., SMITH A. M., 1979. Forage intake and rumination by sheep. $J$. anim. Sci., 49, 1292-1299.

BAE DONG HO, WELCH J. G., SMITH A. M., 1981. Efficiency of mastication in relation to hay intake by cattle. J. anim. Sci., 52, 1371-1375.

BELL F. R., LAWN A. M., 1957. The pattern of rumination behaviour in housed goats. Brit. J. anim. Behav., 5, 85-89.

BLANCHART G., BRUN-BELLUT J., VIGNON B., 1980. Comparaison des caprins aux ovins quant à l'ingestion, la digestibilité et la valeur alimentaire de diverses rations. Reprod. Nutr. Dévelop., 20, 1731-1737.

DEVENDRA C., 1978. The digestive efficiency of goats. World. Rev. anim. Prod., 14, 9-22.

DEVENDRA C., 1981. The utilisation of forages from cassava, pigeon pea, leucaena and groundnut by goats and sheep in Malaysia, 338-346. In MORAND-FEHR P., BOURBOUZE A., de SIMIANE M., Nutrition and systems of goat feeding. Vol. 1. INRA-ITOVIC. Symp. int., Tours, France, 12/15 mai 1981.

DULPHY J. P., RÉMOND B., THÉRIEZ M., 1980. Ingestive behaviour and related activities in ruminants, 103-122. In RUCKEBUSCH Y., THIVEND P., Digestive physiology and metabolism in ruminants, MTP Press Ltd Proceed. 5th int. Symp. on Ruminant physiology, Clermont-Ferrand, $3 / 7$ sept. 1979.

EL-HAG G. A., 1976. A comparison study between desert goats and sheep on efficiency of food utilization. World. Rev. anim. Prod., 12, 43.

FAHMY S. T., NAGA M. A., ABOU-AKKADA A. R., EL-SHAZLY K., 1977. Rumen activities in goats and sheep. Alex. J. agric. Res., 25, 235.

FOCANT M., GALLOUIN F., LECLERCQ M., 1979. Volatile fatty acids and rumination in the goat. Ann. Rech. vét., 10, 226-228.

GEOFFROY F., 1974. Etude comparée du comportement alimentaire et mérycique de deux petits ruminants : la chèvre et le mouton. Ann. Zootech., 23, 63-73.

GIHAD E. A., 1976. Intake, digestibility and nitrogen utilization of tropical natural grass hay by goats and sheep. J. anim. Sci., 43, 879-883.

GIHAD E. A., EL-BEDAWY T. M., ALLAM S. M., 1981. Comparative efficiency of utilization of untreated and $\mathrm{NaOH}$-treated poor-quality roughages through in situ digestion by sheep. goats and buffaloes, 327-337. In MORAND-FEHR P., BOURBOUZE A., de SIMIANE M., Nutrition and systems of goat feeding. Vol. 1. INRA-ITOVIC. Symp. int., Tours, France, $12 / 15$ mai 1981.

GIHAD E. A., EL-BEDAWY T. M., MEHREZ A. Z., 1980. Fiber digestibility by goats and sheep. J. Dairy Sci., 63, 1701-1706.

GORDON J. G., 1958a. The act of rumination. J. agric. Sci., 50, 34-42.

GORDON J. G., 1958b. The effect of time of feeding upon rumination. J. agric. Sci., 51, 81-83.

HUNTINGTON G.B., BRITTON R. A., PRIOR R. L., 1981. Feed intake, rumen fluid volume and turnover, nitrogen and mineral balance and acid-base status of wethers changed from low to high concentrate diets. J. anim. Sci., 52, 1376-1387.

JONES G. M., LARSEN R. E., JAVED A. H., DONEFER E., GAUDREAU J. M., 1972. Voluntary intake and nutrient digestibility of forages by goats and sheep. J. anim. Sci. 34, 830.

METZ J. H. M., 1975. Time patterns of feeding and rumination in domestic cattle. Meded. Landbouwhogeschool, Wageningen, 75-12.

MOHAMED H. H., OWEN E., 1981. Comparison of the maintenance energy requirement of sheep and goats fed dried lucerne or dried grass. In MORAND-FEHR P., BOURBOUZE A., de SIMIANE M., Nutrition and systems of goat feeding. Vol. 2, INRA-ITOVIC. Symp. int., Tours, France, 12/15 mai 1981.

PEARCE G. R., 1965. Rumination in sheep. II. The circadian pattern of rumination. Austr. J. agric. Res., 16, 635-648. 
REMESY C., 1973. Contribution à l'étude de la production et du métabolisme des acides gras volatils chez le rat. Th. Doct., Fac. Sci. Clermont-Ferrand.

RUCKEBUSCH Y., 1963. Recherches sur la régulation centrale du comportement alimentaire chez les ruminants. Th. Doct. Sci., Lyon.

RUCKEBUSCH Y., 1967. Etude polygraphique des variations spontanées ou provoquées du comportement alimentaire chez les petits ruminants. Arch. Sci. physiol, 21, 449.

SCHALK A. F., AMADON R. S., 1928. Physiology of the ruminant stomach (bovine) study of the dynamics factors. Bull. North Dakota agr. exp. Sta., 8, 216.

SCHAMBYE P., PHILLIPSON A. T., 1949. Volatile fatty acids in portal blood of sheep. Nature, 164, 1094-1095.

SIMKINS K. L. Jr., SUTTIE J. W., BAUMGARDT B. R., 1966. Regulation of food intake in ruminants. 3 . Variation in blood and rumen metabolites in relation to food intake. J. Dairy Sci., 48, 1629-1634.

SUDWEEKS E. M., 1977. Chewing time, rumen fermentation and their relationship in steers as affected by diet composition. J. Dairy Sci., 64, 694-701.

UDEN P., 1978. Ph. D. Thesis. Cornell Univ., Ithaca, N.Y., cité par VAN SOEST (1982).

VAN SOEST P. J., 1982. Nutritional ecology of the ruminant. Ruminant metabolism nutritional strategies, the cellulolytic fermentation and the chemistry of forages and plant fibers. Publ. by P. J. VAN SOEST, Cornell Univ., O \& B Books, Corvallis, Oregon 97330 , U.S.A., $374 \mathrm{pp}$.

WAINMAN F. W., 1977. Digestibility and balance in ruminants. Proc. Nutr. Soc., 36, 195.

WELCH J. G., 1982. Rumination, particle size and passage from the rumen. J. anim. Sci., 54, 885-894.

WELCH J. G., SMITH A. M., 1971. Physical stimulation of rumination activity. J. anim. Sci., 33, 1118-1123. 